\title{
Enhancing the Performance of Medium Access Control for WLANs with Multi-beam Access Point
}

\author{
Jianfeng Wang, Student Member, IEEE, Yuguang Fang, Senior Member, IEEE, \\ and Dapeng Wu, Senior Member, IEEE
}

\begin{abstract}
Wireless LANs with multi-beam directional antennas have received intensive attention lately due to the potential gain in throughput performance. However, when the multibeam directional antennas are introduced in this system, the ever popular contention-based medium access control protocol such as IEEE 802.11 MAC is no longer effective, and many challenging problems, such as beam-synchronization problem, beam-overlapping problem, mobility and receiver blocking problem (deafness problem), need to be resolved. In this paper, we propose a novel MAC protocol to carefully address these problems. In addition to improving communication efficiency, we also consider the backward compatibility in our design, whereby an IEEE 802.11 terminal can transparently access a multi-beam access point. Furthermore, we present an analytical model to evaluate the performance of multi-beam wireless LANs. Extensive simulation studies are used to validate the analytical model and show that our scheme can significantly improve the throughput performance.
\end{abstract}

Index Terms-Medium Access Control (MAC), wireless LANs, directional antennas, performance analysis.

\section{INTRODUCTION}

$\mathbf{W}$ ITH the popularity of wireless local access, there is an increasing demand to improve throughput and energy efficiency for data transmission between terminals and an access point. Multi-beam smart antennas bring two major benefits, spatial reuse and antenna gain, both of which are useful in improving the communication efficiency. Therefore, it is of great interest to consider the use of multi-beam smart antennas in a wireless LAN, especially at the access point. The access point is generally more powerful with less physical constraint than mobile terminals (assuming mobile terminals still use omni-antenna), thereby it is feasible to equip multiple smart antennas at the access point. With multi-beam smart antenna system, multiple omni-antenna nodes may transfer data from or to the access-point simultaneously, thus it is potential to increase throughput substantially.

IEEE 802.11 standard provides one mandatory channel access function DCF (Distributed Coordination Function) and one optional channel access function PCF (Point Coordination Function). PCF is centralized, while DCF is fully distributed. In this paper we concentrate on the DCF in our MAC protocol

Manuscript received April 8, 2005; revised October 14, 2005 and March 24, 2006; accepted April 3, 2006. The associate editor coordinating the review of this paper and approving it for publication was M. Zorzi. This work was supported in part by the NSF Career Award ANI-0093241 and under NSF grant DBI-0529012.

The authors are with the Department of Electrical \& Computer Engineering, University of Florida, Gainesville, Florida 32611-6130 (email: fang@ece.ufl.edu).

Digital Object Identifier 10.1109/TWC.2007.05245 design. Obviously, we cannot fully harness the benefits of multi-beam antennas by directly applying the DCF because DCF is a carrier sense based MAC protocol. In such a protocol, once a mobile node is transmitting DATA or RTS to access point, all other nodes in the carrier sensing range should detect the busy channel and defer the channel access. If another node outside the carrier sensing range attempts to access the channel, it may fail even though this node is in an orthogonal beam-sector. The reason is that the DCF follows either 2-way (DATA/ACK) handshake procedure or 4way handshake procedure (RTS/CTS/DATA/ACK), in which a node (including access point) needs to immediately switch between receiving mode and transmitting mode to complete the handshake. However, all beams at the access point can be either in the transmission mode or the receiving mode due to the well-known co-site interference problem, assuming all the beams operate in the same frequency band. Therefore, beamsynchronization (for either transmission or reception) required to facilitate multiple parallel transmissions is hard to achieve. In most cases, only one node can be allowed to transmit at a time. We refer this problem as the beam-synchronization problem.

In addition to the beam-synchronization problem, the beamoverlapping problem, the back-lobe (side-lobe) problem, the multipath rich problem, the mobility issue, the receiver blocking problem and the hidden terminal problem (to be explained later) should be carefully addressed in the MAC design. There is little attention paid to these important issues in the CSMA/CA based MAC protocols for fixed multi-beam wireless LANs or ad hoc networks. Lal et al. [3] provided a performance analysis for simplified CSMA (no handshake and no ACK) in a wireless LAN. However, no detailed solution has been proposed in the paper to address the above challenging problems. Swaminathan et al. [2] addressed the issue of using fixed multi-beam antennas in heterogenous ad hoc networks. A solution based on multiple channels, with one frequency channel serving as control channel and the remaining as data channels, was proposed to address the beam-synchronization problem and the receiver blocking problem. Similarly, the problems due to the imperfection of antennas have not been carefully addressed.

Our system assumes multi-beam access point, omni-antenna mobile nodes and a single frequency channel. The primary goal of our paper is to identify the problems in such a system and propose a distributed MAC layer solution based on IEEE 802.11 DCF to solve the problems with reasonable overhead. Our scheme also considers the backward compatibility with 
IEEE 802.11, whereby an 802.11-based terminal can access a multi-beam access point. To analyze our proposed MAC protocol, we further develop an analytical model to evaluate the performance of multi-beam wireless LANs, another contribution of this paper. It is worth noting that, since all the nodes, including the access point, run under the CSMA/CA-based MAC, our proposed MAC protocol can be easily extended from WLANs to multihop ad hoc networks. For example, in IEEE 802.11 based multihop wireless backhaul networks [4], we believe that the end-to-end throughput will be significantly improved by applying multi-beam antennas at the Transit Access Points (TAP) and our suggested MAC protocol in each node.

The rest of the paper is organized as follows. In the next section, we detail the system model and identify the design challenges. Then we present our proposed protocol for uplink medium access control and downlink medium access control, in Section III and Section IV, respectively. Section V discusses various important issues such as compatibility with IEEE 802.11 terminals, mobility, beam-overlapping, and dense multipath environments. In Section VI, we analytically evaluate the performance of the proposed scheme. Simulation results are provided in Section VII. Finally, we conclude the paper in Section VIII.

\section{System Model And Design Challenges}

\section{A. Antenna System Model}

Two types of multi-beam smart antenna systems have been widely studied in the current literature; one is based on adaptive arrays [5]-[8] and the other is based on the fixedbeam directional antennas [2] [3]. Although adaptive-array based smart antennas may work better in multipath rich environments, it is more complex to design the transceiver and MAC protocol. In this paper, we consider fixed multibeam antenna system due to its simplicity and commercial availability.

Assume the antenna system consists of $M$ sectors, each of which is oriented to provide nonoverlapping $\frac{360^{\circ}}{M}$ azimuth coverage (The number of sectors could be less than $M$ if complete $360^{\circ}$ azimuth coverage is not necessary). Each sector consists of $N$ narrow beams with approximately $\frac{360^{\circ}}{M N}$ beamwidth per beam ${ }^{1}$. One sector is equipped with at least one individual transceiver. At most one user will be selected for communication in each sector at a time. Selection combining is used to switch the strongest narrow beam(s) for a node chosen for communication with access point [1]. The reason we suggest each sector be composed of multiple narrow-beam antennas is as follows. If one sector consists of only one widebeam antenna, the front to back lobe ratio will be low, thus causing significant interference to other sectors. On the other hand, if one sector consists of multiple narrow-beam antennas and the strongest beam is selected for communication each time, the front to back lobe ratio will be high. We can treat multiple narrow-beam antennas in each sector as one logical antenna.

\footnotetext{
${ }^{1}$ The beamwidth of two edge beams at each section may be a little bit larger for better coverage.
}

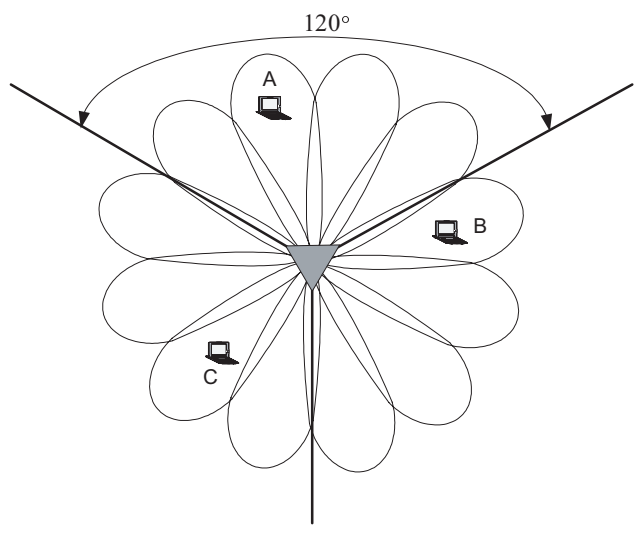

Fig. 1. An example of sectorized multi-beam antenna system.

Since narrow beam is used in our system, the front-to-back and the front-to-side gain ratios can be high. Fig. 1 illustrates a 3-sector 12-beam directional antenna system; each sector consists of four beams (with approximately $30^{\circ}$ beamwidth per beam) and provides $120^{\circ}$ azimuth coverage; the front-toback ratios for the beams can be as high as 20-35 dB [1].

\section{B. Design Challenges}

As we mentioned early in this paper, the first challenging problem is the beam-synchronization problem. To maximally exploit the spatial reuse, all the nodes chosen for communication (one in each sector) should be synchronized to transmit (receive) DATA in one phase and receive (transmit) ACK in another phase. Apparently, DCF was not originally designed to enable such a coordinated function. The design challenge here is to provide a more coordinated function than DCF to facilitate the concurrent use of multiple antenna beams, while still maintaining the ad hoc nature.

The second challenge is to tackle the beam-overlapping problem, the back-lobe (side-lobe) problem and the multipath rich problem, due to the imperfection of directional antennas. Small portion of beam-overlapping area generally exists for two adjacent beams. Once a node located in the overlapping area intends to send data (to the access point) or receive data (from the access point), the intended transmission or reception of any other node (in either one of two adjacent beams) will collide with the one in the overlapping area. In addition, even if using high-gain narrow-beam directional antennas in our system, the side-lobe and back-lobe effects cannot be totally ignored. If a node is too close to access point, it can still fall in the side-lobe or back-lobe of many other beams. Moreover, a node located in multipath rich environments may hear transmissions from each beam from the access point and each beam at an access point can hear transmission from the same mobile terminal. Thus, all these three problems should be addressed in the MAC design.

The third challenge is to solve the receiver blocking problem and the hidden terminal problem. We refer to Fig. 1 for illustration. Suppose the access point directs a beam to node A for downlink data transmission (without informing nodes in other beam-sectors). If node $\mathrm{A}$ is far from node $\mathrm{C}$ and the CTS of node A cannot reach $\mathrm{C}$, node $\mathrm{C}$ is not aware of the transmission from the access point to node A. Now 
node $\mathrm{C}$ attempts to communicate with the access point. Since the access point cannot hear the RTS from node $\mathrm{C}$ during its transmission to node A, node C may keep sending RTS for many times without getting response from the access point (receiver side). We refer this problem as the receiver blocking problem or deafness problem. The receiver blocking problem will waste energy and network capacity as a result of network nodes engaging in the repeated, unproductive transmissions. Besides the receiver blocking problem, the hidden terminal problem also occurs. If node $\mathrm{C}$ is close to node $\mathrm{A}$, node C's transmission may corrupt A's reception of data from the access point.

Yet another challenge is to deal with the mobility. Mobility may not be a big problem for traditional access point with omni-antenna. However, it arises as a non-negligible problem for access point with multi-beam antennas, in which each node is physically associated with a beam-sector. As a mobile node moves from one beam-sector to another, medium access control, especially the downlink medium access control will be affected. An efficient location updating algorithm (joint design with MAC) should be provided to keep the freshness of location information with reasonable overhead.

Finally, we need to provide the backward compatibility with IEEE 802.11 standard. Backward compatibility maintains the inter-operability between the legacy systems and the new systems, and increases the utilization of scarce wireless spectrum resource. Thus it is desirable to maintain the compatibility, especially with 802.11 DCF based WLANs, which have already been widely deployed.

\section{Uplink Medium AcCess Control}

\section{A. Framework}

The basic idea of our MAC protocol is to introduce a timing-structure to facilitate multiple handshakes (sequential or overlapping) before parallel collision-free data transmissions. Our proposed uplink MAC structure is shown in Fig. 2. The uplink medium access is initiated by an access point. Upon receiving a ready-to-receive(RTR) message sent by an access point, all the nodes having uplink packets in the queues contend for the channel access during the contention period $T_{1}$ shown in Fig. 2. The contention resolution scheme we design will facilitate multiple nodes to win out and the winning nodes will be collision-free with each other. Here by "collisionfree", we mean the winning nodes will not collide with each other when they simultaneously send DATA to or receive ACK from the access point in the period of $T_{2}$ and $T_{3}$, respectively. In order to be collision-free, different winning nodes should be in different beam-sectors. In the phase of DATA transmission, multiple back-to-back packets are allowed to transmit by one node as long as the total time of transmitting these multiple packets does not exceed the length of $T_{2}$. Note that power control and/or auto rate scheme can be incorporated in our scheme to further increase the throughput and energy efficiency. In the period of parallel ACKs, the access point will transmit one ACK for each user concurrently because the users are in different sectors.

The access point initiates the uplink medium access control adaptive to the current traffic load. Since the access point also

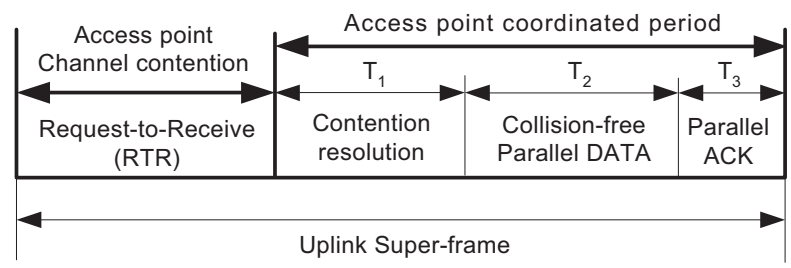

Fig. 2. Uplink super-frame.

runs in the ad hoc mode, the access point needs to contend for channel as an ordinary node ${ }^{2}$. However, the access point can send RTR messages in a higher priority, i.e., with shorter Interframe-space (IFS) and contention window size, over mobileterminals to exchange RTS messages. We assume the access point still follows the de-facto Exponential-Backoff algorithm to resolve collision with other ordinary nodes. Thanks to the smaller Inter-frame-space (IFS) and contention window size, it will be much easier for the access point to capture the channel than mobile terminals. This priority differentiation guarantees that the access point gains a higher priority to access the channel, which is typically desirable.

\section{B. Channel Contention}

In this section, we describe the channel contention scheme used in the period $T_{1}$, which is based on the CSMA/CA. Specifically, the CSMA-based RTS/CTS exchange is illustrated in Fig. 3. The time of an idle slot $T_{i d}$, the time of collision $T_{c o l}$, and the time spent for a successful contention $T_{\text {suc }}$ are defined as

$$
\left\{\begin{array}{l}
T_{i d}=\sigma \\
T_{c o l}=R T S+D I F S \\
T_{s u c}=R T S+S I F S+C T S+S I F S
\end{array}\right.
$$

where $\sigma$ is the length of a time slot. Different from the traditional CSMA with omni-antenna access point, two nodes simultaneously transmitting RTS to the access point may not collide with each other if they are in different beam-sectors. In other words, if there is only one node sending RTS in a given beam-sector and it is free of beam-overlapping problem and multipath problem to be explained later, the node succeeds no matter how many nodes concurrently sending RTSs in other beam-sectors. Unless each beam-sector has collisions of RTSs, the access point will send CTSs in all beam-sectors concurrently after receiving RTSs. Specifically, for a beamsector, in which one RTS is correctly received, the access point will reply with a positive CTS to confirm that the node sending RTS can transmit DATA in period $T_{2}$; all the other intended transmitting nodes in the beam-sector will then keep silent until the end of the current access-point-coordinated-period. For a beam-sector experiencing collisions, the access point will reply with a negative CTS to indicate that the contention fails; all the intended transmitting nodes in the unsuccessful beam-sector, including those failed nodes, will continue to contend for channel until one node wins out or period $T_{1}$ ends. For a beam-sector, in which there is no RTS, the access point will also send CTS, to silent intended transmitting

\footnotetext{
${ }^{2}$ The access point will keep silent in all beam-sectors if it senses the channel is busy.
} 


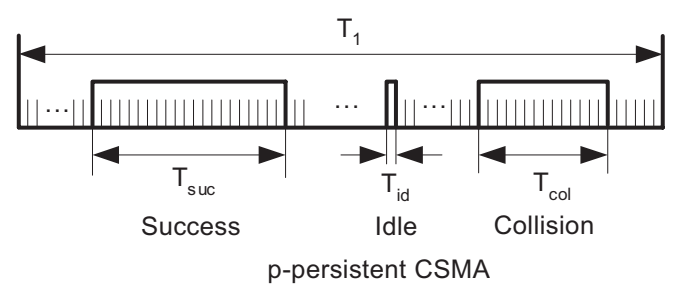

Fig. 3. CSMA based contention resolution scheme for baseline MAC.

nodes in the beam-sector for a while, thereby mitigating the hidden terminal problem and the receiver-blocking problem; the intended transmitting nodes in a beam-sector with no RTS being sent, will continue to contend for channel after receiving the CTS, until one node wins out or period $T_{1}$ ends. If each beam-sector has collisions of RTSs, the access point may just keep silent. All intended transmitting nodes including those which sent the RTSs will resume contention after the channel is idle for a period of DIFS.

It is possible that RTS receptions are not simultaneous, but overlapping. For this case, after receiving the first correct RTS, the access point immediately run the same procedure as if only one correct RTS is received (introduced above). For simplicity, the second RTS, no matter whether it is correctly received or not, will be ignored.

To resolve the contention among the intended transmitting nodes during period $T_{1}$, either backoff-based algorithm or $p$-persistent algorithm can be used. To achieve the optimal performance, the contention window size $(\mathrm{CW})$ for the backoff algorithm or the medium access probability $p$ for the $p$ persistent algorithm should be adjusted based on the number of active users in each beam-sector.

It can be seen that the schemes described above try to allow as many independent beam-sectors to be utilized for parallel transmission as possible with the constraints of the time-limit, $T_{1}$, for channel contention. Assume $T_{2}$ and $T_{3}$ are fixed. The factors that affect the overall system utilization are $p$ or $\mathrm{CW}$, the length of $T_{1}$ and the time duration between the end of the current super-frame and the beginning of its next super-frame, which is denoted as $T_{\text {int }}$. Note that $\mathrm{CW}$ can be approximated by $1 / p$. Hence, without loss of generality, we focus on the parameter $p$ instead of CW. To achieve the optimal performance, we design methods to adjust $p, T_{1}$ and $T_{\text {int }}$, which are presented in the next section.

\section{Online Adapting System Parameters}

This section discusses how the access point adapts the system parameters introduced in Section III-B, which include $p, T_{1}$ and $T_{i n t}$, to the system dynamics. The parameters are updated iteratively at the end of each super-frame. At the beginning of next super-frame, updated $p$ and $T_{1}$ will be included in the RTR message to inform each node. We follow the stochastic approximation approach [12] to adjust system parameters, assuming that the number of competing nodes in each beam-sector does not change very fast.

Let $k, \frac{1}{W^{k}}, \bar{L}_{m}^{k}$ and $\beta_{1}$ denote the super-frame index, the updating step-size and the exponentially weighted aggregate queue length in beam-sector $m$ (the queue length information is fed back from mobile terminals to the access point via RTS messages) and the scaling factor, respectively. As before, $M$ is the total number of beam-sectors. We adjust $T_{\text {int }}$ by

$$
\left\{\begin{array}{l}
T_{\mathrm{int}}^{k+1}=\bar{T}_{\mathrm{int}}^{k}-\frac{\beta_{1}}{W^{k}}\left(\bar{L}_{\max }^{k}-L_{\phi}\right) \\
\bar{T}_{\mathrm{int}}^{k}=\left(1-\frac{1}{W^{k}}\right) \bar{T}_{\mathrm{int}}^{k-1}+\frac{1}{W^{k}} T_{\mathrm{int}}^{k} \\
\bar{L}_{\max }^{k}=\operatorname{Max}\left\{\bar{L}_{m}^{k}: 1 \leq m \leq M\right. \\
\bar{L}_{m}^{k}=\left(1-\frac{1}{W^{k}}\right) \bar{L}_{m}^{k-1}+\frac{1}{W^{k}} L_{m}^{k}
\end{array}\right.
$$

such that each super-frame can be fully utilized while the average aggregate queue length in any beam-sector will not significantly exceed the system-targeted value $L_{\phi}$.

Denote $N_{c o l, m}^{k}$ as the number of collisions observed at beam-sector $m$ in super-frame $k$. Denote $\beta_{2}$ as the scaling factor and $\beta_{3}$ as the penalty price of a collision. Medium access probability in each beam-sector, $p_{m}$, is updated according to

$$
\left\{\begin{array}{l}
p_{m}^{k+1}=\bar{p}_{m}^{k}+\frac{\beta_{2}}{W^{k}}\left(1-\beta_{3} N_{c o l, m}^{k}\right) \\
\bar{p}_{m}^{k}=\left(1-\frac{1}{W^{k}}\right) \bar{p}_{m}^{k-1}+\frac{1}{W^{k}} p_{m}^{k}
\end{array},\right.
$$

in which we increase the medium access probability by one step-size when idle events or success events are observed and decrease the medium access probability by multiple step-size when collisions are observed.

We set $T_{1}$ a little bit greater than the total average time required to resolve a user per active beam-sector(we call a beam-sector as active if the estimated number of competing nodes in that beam-sector is greater than a certain value). The average time to resolve a collision, denoted as $\tilde{T}(n, p)$, can be calculated as

$$
\tilde{T}(n, p)=T_{s u c}+\frac{p_{c o l}}{p_{s u c}} T_{c o l}+\frac{p_{i d}}{p_{s u c}} T_{i d},
$$

where $p_{i d}$ is the probability of an idle event, $p_{c o l}$ is the probability of a collision event and $p_{s u c}$ is the probability of a success (successful-contention) event, respectively, in $p$ persistent CSMA. Given the medium access probability $p$ and the number of competing nodes $n, p_{i d}, p_{c o l}$ and $p_{s u c}$ are computed, respectively, as follows:

$$
\left\{\begin{array}{l}
p_{\text {suc }}=n p(1-p)^{n-1} \\
p_{i d}=(1-p)^{n} \\
p_{\text {col }}=1-p_{\text {suc }}-p_{i d}
\end{array} .\right.
$$

To estimate the number of competing nodes, we use the simple ARMA (Auto Regressive Moving Average) run-time estimation algorithm in our paper (we believe better performance could be achieved by more advanced estimation algorithms [9]). As shown in Eq. 5, once we know the medium access probability used and the idle probability observed, we can get the estimated number of competing nodes. Denote $\bar{p}_{i d, m}^{k}$ as the exponentially averaged idle probability observed in beam-sector $m$ before super-frame $k+1 . \hat{n}_{m}^{k}$ and $\bar{p}_{i d, m}^{k}$ can be derived as follows

$$
\left\{\begin{array}{l}
\hat{n}_{m}^{k+1}=\log _{1-\bar{p}_{m}^{k}}\left(\bar{p}_{i d, m}^{k}\right) \\
\bar{p}_{i d, m}^{k}=\left(1-\frac{1}{W^{k}}\right) \bar{p}_{i d, m}^{k-1}+\frac{1}{W^{k}} \hat{p}_{i d, m}^{k}
\end{array}\right.
$$

where $\hat{p}_{i d, m}^{k}$ is the idle probability observed in beam-sector $m$ at super-frame $k$.

Now, we are ready to calculate $T_{1}$ as

$$
T_{1}^{k}=\beta_{4} \sum_{m} \kappa_{m} \tilde{T}\left(\hat{n}_{m}^{k}, p_{m}^{k}\right)
$$


where $\beta_{4}$ is a constant slightly greater than 1 , and $\kappa_{m}$ is calculated below

$$
\kappa_{m}= \begin{cases}1 & \hat{n}_{m}^{k} \geq 0.5 \\ 0 & \hat{n}_{m}^{k}<0.5\end{cases}
$$

So far we assume we can choose appropriate $T_{i n t}, p$ and $T_{1}$ according to the feedback of the backlog queue length, the observation of collision events and the estimation of the number of competing nodes. We notice that the choice of the updating step-size $\frac{1}{W^{k}}$ is important for system stability and convergence speed. A larger step size is more responsive to change but leads to higher fluctuation in stochastic approximation process. On the other hand, a smaller step size can keep stochastic approximation more smooth, but is less responsive to change. To maintain the balance between the convergence speed and the convergence smoothness, we take $W^{k}$ as

$$
W^{k}= \begin{cases}k, & k<W_{\max } \\ W_{\max }, & k \geq W_{\max }\end{cases}
$$

where $W_{\max }$ is the updating window size.

\section{Downlink Medium Access Control}

In this section, we describe downlink medium access control. Our proposed downlink MAC structure is shown in Fig. 4. Since the access point runs in the ad hoc mode, the access point needs to send RTS message to contend for channel to deliver downlink data. Similar to the RTR message used in the uplink medium access control, RTS message by the access point can be sent at a higher priority, i.e., with shorter Interframe-space (IFS) and contention window size, over mobileterminals to exchange RTS messages.

We examine two network scenarios: static and mobile. In the static scenario, we assume the access point knows which beam-sector each node is located at; the location information could be obtained during the association process. In the mobile scenario, we assume that the access point keeps the cached beam-location information of each node, which could be updated reactively whenever there is data needed to be exchanged between the access point and a given node or could be updated proactively using the periodic polling/probing initiated by the access point. The accuracy of the cached location information depends on the update frequency and the mobility pattern of a given node.

Based on the location information, the access point will choose multiple intended receivers, each of which is supposed to be associated with a different beam-sector, to facilitate multiple collision-free concurrent data transmissions. After choosing candidate receivers, the access point concurrently sends multiple RTSs to multiple beam-sectors, each of which contains the MAC-address of the corresponding candidate receiver. If the cached location information is accurate, i.e., the node is still in the expected beam-sector, the candidate receiver will reply with CTS immediately upon receiving RTS. Note that multiple CTSs may transmit concurrently if the cached location information is accurate; these CTSs will not collide with each other because they are in different beam-sectors. If the cached beam-location information of an intended receiver is not accurate, the access point will not receive CTS from the intended receiver; then, the access point will disable

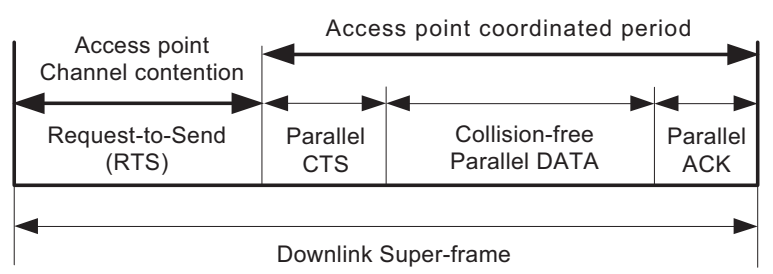

Fig. 4. Downlink super-frame.

the cached information, and poll the out-of-date node omnidirectionally during the period between the end of the current super-frame and the beginning of the next super-frame. Note that there exists a period between the end of the current superframe and the beginning of the next super-frame.

Upon receiving CTSs from candidate receivers, the access point will immediately initiate downlink parallel DATA transmissions to those candidate receivers. Similar to the uplink case, rate-adaptation, power control and multiple-packet transmission could be incorporated in the downlink DATA transmission and the accumulated ACK is used to acknowledge the received multiple data packets.

\section{Discussions}

\section{A. Compatibility}

Since 802.11 DCF-based WLANs have been widely deployed, the question is how an IEEE 802.11 node transmits and receives data to/from a multi-beam access point. We first discuss the downlink medium access. For the downlink medium access, one can easily see that no extra modification is needed for our proposed scheme to maintain compatibility if unicast-directional RTS and Parallel-CTS (shown in Fig. 4) are used, because the procedure strictly follows the operation of IEEE 802.11 standard. For the uplink medium access, there is a difference between IEEE 802.11 MAC and our proposed scheme; IEEE 802.11 node always follows the sender-initiated medium-access rule while in our scheme, uplink medium access is access-point initiated, i.e., receiver-initiated. Fortunately, a multi-beam access point can immediately identify that a node uses the IEEE 802.11 MAC if the node transmits an RTS not in the uplink super-frame; upon receiving such an RTS, the access point will follow the procedure of IEEE 802.11 standard and immediately reply with a CTS. Hence, the whole process is transparent to the IEEE 802.11 node.

\section{B. Mobility}

Since the beam-location information cached in the access point may not be accurate when nodes move, "mis-hit" could happen in the downlink medium access. The probability of "mis-hit" gets higher with the increase of mobility. Our scheme takes both proactive and reactive mechanisms to minimize the negative effects. The access point may reactively poll a node omni-directionally to get updated beam-location information whenever "mis-hit" occurs. In addition, once a node intends to access the medium in the uplink, it will automatically update the cached beam-location information. For the case of high node mobility, the reactive mechanisms explained above may not be enough; to obtain updated beamlocation information, we modify the MAC operation in Section IV as follows. The access point places the MAC addresses 
of all the intended receivers in one RTS, sends the RTS in all beam-sectors, and requests each intended node to reply with a CTS one after another to report its current beam-location information and channel state. In other words, as shown in Fig. 4, unicast-directional-RTS will be changed to multicastomnidirectional-RTS, and Parallel-CTS will be changed to Sequential-CTS.

\section{Beam-Overlapping Problem}

Since we only allow one node to communicate with the access point in one sector, beam-overlapping problem can be limited to the boundary area between two adjacent sectors. Our proposed MAC is aware of overlapping problem and will take precaution to avoid the overlapping problem in both uplink medium access and downlink medium access.

In the uplink medium access, whenever a node located in the overlapping area sends RTS successfully, the access point may receive two copies of RTS from the two adjacent beams. The access point can identify whether the overlapping problem occurs by comparing the sender's MAC-address of these two RTSs. Then the access point will send one CTS to each beamsector sequentially (the second CTS is SIFS after the first CTS) to confirm the RTS and prevent other nodes in both beam-sectors from further contending for the channel, thereby guaranteeing that the data transmission will be collision-free. Note the nodes located in the overlapping area (including the one sending RTS) may receive two copies of CTS sequentially and will ignore the second one without causing incorrect MAC operations. Once the access point receives DATA (actually two copies), the MAC layer at the access point will convey one correct copy to the upper layer.

As mentioned in section III-B, our protocol allows two beam-sectors to send two distinct CTSs in parallel, one CTS per beam-sector, to two distinct nodes if the access point simultaneously receives two RTSs correctly from these two nodes. If these beam-sectors are neighbors, those nodes in the overlapping area may not correctly receive either CTS. Thus they cannot set NAV correctly and may contend for channel later even though the channel has been reserved by previous winning nodes. If this is the case, the access point will send a negative CTS to the node sending RTS if the corresponding beam-sector has already been reserved. Thus, it still guarantees the correct protocol operation.

In the downlink medium access, the access point will not allow other nodes in two adjacent beams(with an overlapping area) to receive DATA if a node located in the overlapping area has already been chosen for reception. We can use the following procedure to identify whether a node is located in an overlapping area. First, the access point detects an overlapping area by certain mechanism, e.g., two copies of RTSs from two adjacent beams have the same MAC address of the sender. Then, the cached beam-location about a node can help the access point to identify whether the node is located in the overlapping area.

\section{Multipath Rich Problem and Back-Lobe Problem}

A node located in multipath rich environments or located too close to access point (back-lobe problem) may hear transmissions from multiple beam-sectors; vice versa, multiple beam-sectors at the access point can hear transmission from the same mobile terminal. In this case, it is better for the access point to run in the omnidirectional transmission/reception mode as in IEEE 802.11 access point. The approach to addressing the multipath rich problem and back-lobe problem is similar to addressing the beam-overlapping problem. We describe the approach as below.

In the uplink medium access, whenever a node located in a multipath rich area sends RTS successfully, the access point may receive multiple copies of RTS from multiple beamsectors. The access point can identify whether the multipath rich problem occurs by comparing the sender's MAC addresses of these RTSs.

In the downlink medium access, the access point will not allow other nodes to receive DATA if a node located in a multipath rich area is already chosen for reception. We can use the following procedure to identify whether a node is located in a multipath rich area. First, the access point detects a multipath rich area by certain mechanism, e.g., multiple copies of RTSs from multiple beam-sectors have the same MAC address of the sender. Then, the cached beam-location about a node can help the access point to identify whether the node is located in the multipath rich area.

It is worthy to point out that, since we use the same strategy to detect and solve the beam-overlapping problem, the multipath rich problem and the back-lobe problem, we do not really need to distinguish these problems from one to another. Instead, we can abstract all these problems as the imperfect beam-forming ${ }^{3}$ problem and follow the same procedure as introduced above to solve the problem.

\section{Performance AnAlysis}

In this section, we analyze the performance of the system operating in the saturated state, i.e., the traffic source always has packets to send. Suppose the system has $M$ disjoint beam-sectors, each of which has $n$ users. The access point always has packets in the queue for transmission to each node, and each node always has packets for transmission to the access point. This performance analysis gives us a performance bound we can achieve.

According to our protocol, both downlink medium access and uplink medium access are coordinated by the access point. Denote the ratio of the uplink super-frame frequency to the downlink super-frame frequency is represented as $\psi /(1-$ $\psi)(0 \leq \psi \leq 1)$. Denote $S_{u}$ as the pure uplink saturated throughput (the throughput under the condition that uplink traffic is saturated and there is no downlink traffic) and $S_{d}$ as the pure downlink saturated throughput (the throughput under the condition that downlink traffic is saturated and there is no uplink traffic). The total throughput can be represented as

$$
S=\psi S_{u}+(1-\psi) S_{d}
$$

Since it is easy to achieve $S_{d}$, we focus on the derivation of $S_{u}$ as below.

\footnotetext{
${ }^{3}$ The beam-forming discussed throughout our paper is fixed beam-forming, especially switched beam-forming, not adaptive beam-forming.
} 

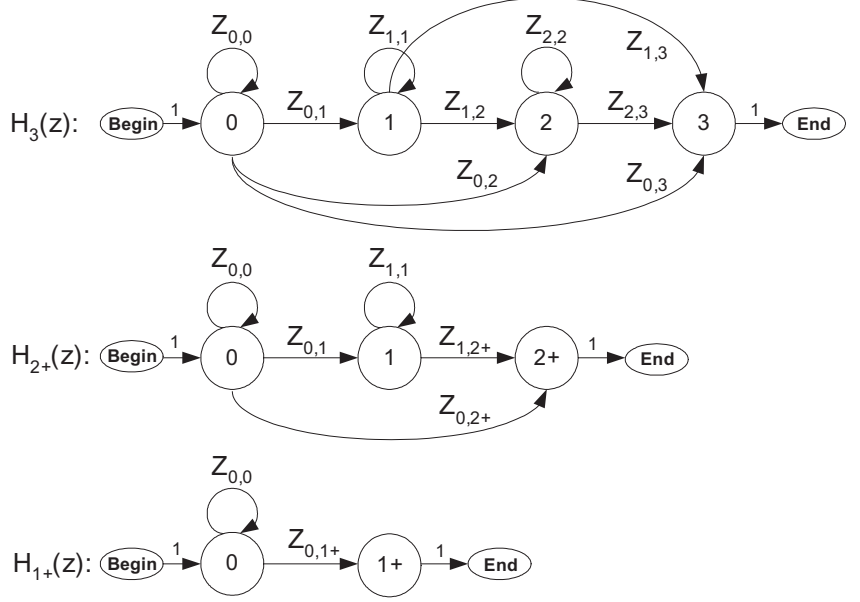

Fig. 5. State transition diagram for CSMA based contention resolution process.

We illustrate our derivation of $S_{u}$ in the case where $M=3$ (the same approach can be applied to derive $S_{u}$ for arbitrary number of beam-sectors). Assume each beam-sector has $n$ nodes and each node always has packets waiting for transmission to the access point. We assume $p$-persistent CSMA is used for collision resolution in contention period $T_{1}$. The probability of medium access for each user in $p$-persistent CSMA is $p$. Assume each user uses the basic rate to send all the control messages. Denote $P_{i}$ the probability that there are only $i$ users winning the channel after contention period $T_{1}$ in a super-frame (note that the maximal number of winning users equals to the number of beam-sectors). Denote $l$ the DATA packet size. Now we can obtain the total throughput as

$$
S_{d}=\frac{\sum_{i=1}^{3} P_{i} \times i \times l}{R T R+T_{1}+T_{2}+T_{3}}
$$

Now the key to calculate the throughput is to find $P_{1}, P_{2}$ and $P_{3}$. Let us start with $P_{3}$. Consider a $p$-persistent CSMA process which starts from a reference time and ends when 3 nodes (each one is located in a different beam-sector) win out; denote $T_{s}^{(3)}$ the total service time ${ }^{4}$ to allow 3 nodes to win out. $T_{s}^{(3)}$ is a random variable and the probability that $T_{s}^{(3)}$ equals to $i \mu \mathrm{s}$ is $\operatorname{Pr}\left(T_{s}^{(3)}=i\right)$. Thus the total probability that $T_{s}^{(3)}$ is less than $T_{1}$, i.e., $P_{3}$, is $\sum_{0}^{T_{1}} \operatorname{Pr}\left(T_{s}^{(3)}=i\right)$. To obtain the probability distribution of $T_{s}^{(3)}$, we apply the probability generating function (PGF) $H_{3}(z)$, which is the $z$-transform of the probability mass function of $T_{s}^{(3)} . H_{3}(z)$ and $T_{s}^{(3)}$ have the relation as $H_{3}(z)=\sum_{i=0}^{\infty} \operatorname{Pr}\left(T_{s}^{(3)}=i\right) z^{i} \cdot H_{3}(z)$ is derived based on the state transition diagram for the $p$ persistent CSMA process that starts from a reference time and ends when 3 nodes win out, as shown in Fig. 5.

To obtain $P_{2}$, we consider a p-persistent CSMA process which starts from a reference time and ends when at least 2 nodes (each one is located in a different beam-sector) win out. denote $T_{s}^{(2+)}$ the total service time to allow at least 2 nodes to win out. $T_{s}^{(2+)}$ is a random variable and the probability that $T_{s}^{(2+)}$ equals to $i \mu \mathrm{s}$ is $\operatorname{Pr}\left(T_{s}^{(2+)}=i\right)$.
Thus, the total probability that $T_{s}^{(2+)}$ is less than $T_{1}$, is $\sum_{0}^{T_{1}} \operatorname{Pr}\left(T_{s}^{(2+)}=i\right)$. Hence, $P_{2}=\sum_{0}^{T_{1}} \operatorname{Pr}\left(T_{s}^{(2+)}=i\right)-$ $P_{3}$. To obtain the probability mass function of $T_{s}^{(2+)}$, we need the probability generating function $H_{2+}(z)$, where $H_{2+}(z)$ and $T_{s}^{(2+)}$ are related by $H_{2+}(z)=\sum_{i=0}^{\infty} \operatorname{Pr}\left(T_{s}^{(2+)}=i\right) z^{i}$. $H_{2+}(z)$ is derived from the state transition diagram for the $p$-persistent CSMA process that starts from a reference time and ends when at least 2 nodes win out, as shown in Fig. 5.

Following the similar definition and derivation process as $P_{2}$, we can obtain $H_{1+}(z)=\sum_{i=0}^{\infty} \operatorname{Pr}\left(T_{s}^{(1+)}=i\right) z^{i}$ and $P_{1}=$ $\sum_{0}^{T_{1}} \operatorname{Pr}\left(T_{s}^{(1+)}=i\right)-P_{2}-P_{3}$. In summary, we obtain

$$
\left\{\begin{array}{l}
P_{3}=\sum_{0}^{T_{1}} \operatorname{Pr}\left(T_{s}^{(3)}=i\right) \\
P_{2}=\sum_{0}^{T_{1}} \operatorname{Pr}\left(T_{s}^{(2+)}=i\right)-P_{3} \\
P_{1}=\sum_{0}^{T_{1}} \operatorname{Pr}\left(T_{s}^{(1+)}=i\right)-P_{2}-P_{3}
\end{array}\right.
$$

and

$$
\left\{\begin{array}{l}
H_{3}(z)=\sum_{i=0}^{\infty} \operatorname{Pr}\left(T_{s}^{(3)}=i\right) z^{i} \\
H_{2+}(z)=\sum_{i=0}^{\infty} \operatorname{Pr}\left(T_{s}^{(2+)}=i\right) z^{i} \\
H_{1+}(z)=\sum_{i=0}^{\infty} \operatorname{Pr}\left(T_{s}^{(1+)}=i\right) z^{i} .
\end{array}\right.
$$

Next, we study the state transition diagrams of $H_{3}(z)$, $H_{2+}(z)$ and $H_{1+}(z)$, shown in the Fig. 5. In the state transition diagram of $H_{3}(z)$, there are 4 states (except the begin state and the end state); the index of each state represents the number of successful winning nodes. In each state, three events may happen, i.e., idle, collision or success. If the event is idle, the state keeps the same after $T_{i d}(\mu s)$ time elapses; the probability of idle event is $P_{i d}^{(i, i)}$, where $i$ is the index of the state. If the event is collision, the state also keeps the same after $T_{\text {col }}(\mu s)$ time elapses; the probability of collision event is $P_{c o l}^{(i, i)}$, where $i$ is the index of the state. Similarly, if the event is a success, the state changes to a state with a larger index after $T_{\text {suc }}(\mu s)$ time elapses; the probability of a success event is $P_{s u c}^{(i, j)}$, where $i$ and $j$ are the index of states before and after transition, respectively.

In the state transition diagram of $H_{2+}(z)$ and $H_{1+}(z)$, the state $2+$ and $1+$ indicate that there are at least 2 winning nodes and at least 1 winning node, respectively. Denote the transition probability from state $i$ to state $j+(i<j<3)$ as $P_{s u c}^{(i, j+)}$. Denote the state transition function in the $z$-transform domain for each branch of state transition as $Z_{i, i}, Z_{i, j}$ and $Z_{i, j+}$, as shown in Fig. 5. Based on the well-known Mason formula [10] [11], we obtain $H_{1+}(z), H_{2+}(z)$ and $H_{3}(z)$ as follows

$$
\left\{\begin{array}{l}
H_{1+}(z)=\frac{Z_{0,1+}}{1-Z_{0,0}} \\
H_{2+}(z)=\frac{Z_{0,2}}{1-Z_{0,0}}+\frac{Z_{0,1} Z_{1,2+}}{\left(1-Z_{0,0}\left(1-Z_{1,1}\right)\right.} \\
H_{3}(z)=\frac{Z_{0,3}}{1-Z_{0,0}}+\frac{Z_{0,2} Z_{2,3}}{\left(1-Z_{0,0}\left(1-Z_{2,2}\right)\right.}+\frac{Z_{0,1} Z_{1,3}}{\left(1-Z_{0,0}\right)\left(1-Z_{1,1}\right)} \\
\quad+\frac{Z_{0,1} Z_{1,2} Z_{2,3}}{\left(1-Z_{0,0}\right)\left(1-Z_{1,1}\right)\left(1-Z_{2,2}\right)}
\end{array}\right.
$$


TABLE I

SIMULATION PARAMETERS

\begin{tabular}{|c|c|c|c|}
\hline Parameter & Value & Parameter & Value \\
\hline \hline Basic rate & $2($ Mbits/s $)$ & Packet Size $(l)$ & $1000($ bytes $)$ \\
\hline PHY header & $192(\mu \mathrm{s})$ & $T_{2}$ & $4000(\mu \mathrm{s})$ \\
\hline$\sigma$ & $20(\mu \mathrm{s})$ & $T_{3}$ & $258(\mu \mathrm{s})$ \\
\hline SIFS & $10(\mu \mathrm{s})$ & $L_{\phi}$ & 1 \\
\hline DIFS & $40(\mu \mathrm{s})$ & $\beta_{1}$ & 0.05 \\
\hline RTS & $168($ bits $)$ & $\beta_{2}$ & 0.1 \\
\hline CTS & $136($ bits $)$ & $\beta_{3}$ & 3 \\
\hline RTR & $96($ bits $)$ & $\beta_{4}$ & 1.1 \\
\hline ACK & $112($ bits $)$ & $W_{\max }$ & 200 \\
\hline
\end{tabular}

According to the transition time and the transition probability, we take the $z$-transform and obtain

$$
\left\{\begin{array}{lr}
Z_{i, i}=P_{i d}^{(i, i)} z^{T_{i d}}+P_{\text {col }}^{(i, i)} z^{T_{\text {col }}}, & 0 \leq i<3 \\
Z_{i, j}=P_{\text {suc }}^{(i, j)} z^{T_{\text {suc }}}, & 0 \leq i<j \leq 3 \\
Z_{i, j+}=P_{\text {suc }}^{(i, j+)} z^{T_{\text {suc }}}, & 0 \leq i<j<3 .
\end{array}\right.
$$

Now we derive $P_{i d}^{(i, i)}, P_{c o l}^{(i, i)}, P_{\text {suc }}^{(i, j)}$ and $P_{\text {suc }}^{(i, j+)}$. Consider that there are $n$ nodes in each beam-sector and the probability of medium access for each user at the beginning of an idle slot is $p$. Recall that two nodes will not collide with each other for a given beam-sector if there is only one user to send RTS in that beam-sector and the probability that there is only one user to send RTS in that beam-sector is $P_{s}=n p(1-p)^{n-1}$. Then, we have

$$
\left\{\begin{array}{lr}
P_{i d}^{(i, i)}=(1-p)^{(3-i) n}, & 0 \leq i<3 \\
P_{s u c}^{(i, j)}=\left(\begin{array}{c}
3-i \\
j-i
\end{array}\right) P_{s}^{j-i}\left(1-P_{s}\right)^{3-j}, & 0 \leq i<j \leq 3 \\
P_{\text {col }}^{(i, i)}=1-P_{i d}^{(i, i)}-\sum_{j=i+1}^{3} P_{\text {suc }}^{(i, j)}, & 0 \leq i<3 \\
P_{\text {suc }}^{(i, j+)}=\sum_{k=j}^{3} P_{\text {suc }}^{(i, k)}, & 0 \leq i<j<3 .
\end{array}\right.
$$

In summary, from Eq. (16), (15) and (14), we obtain $H_{1+}(z), H_{2+}(z)$ and $H_{3}(z)$. Based on $H_{1+}(z), H_{2+}(z)$, $H_{3}(z)$, and Eqs. (13) and (12), we can calculate $P_{1}, P_{2}$ and $P_{3}$. Based on $P_{1}, P_{2}, P_{3}$ and Eq. 11, we can finally compute $S_{u}$.

Using the same approach presented above, we can derive $S_{u}$ for arbitrary number of beam-sectors, in each of which there are $n$ nodes and the $p$-persistent CSMA is used for uplink channel contention.

\section{Simulation Results}

We base our simulation on ns2. The default system parameters are shown in Table I. All packets are sent at 2Mbit/sec. Two-ray ground channel model is used in our simulation.

We first examine the ideal saturated case, which provides a performance limit we can achieve for uplink medium-access. We assume beam-forming is perfect and there is no beamoverlapping problem and the multipath rich problem. Nodes are evenly distributed in different beam-sectors and all traffic goes from nodes to the access point. We set the medium access probability $p$ as $\frac{1}{2 n}$. $T_{1}$ is set to $700 M \mu \mathrm{s}$. $T_{\text {int }}$ is set to 0 . As shown in Fig. 6, our scheme can achieve 1.59, 2.33 and 2.96 times of the 802.11 throughput, when the beam-sector

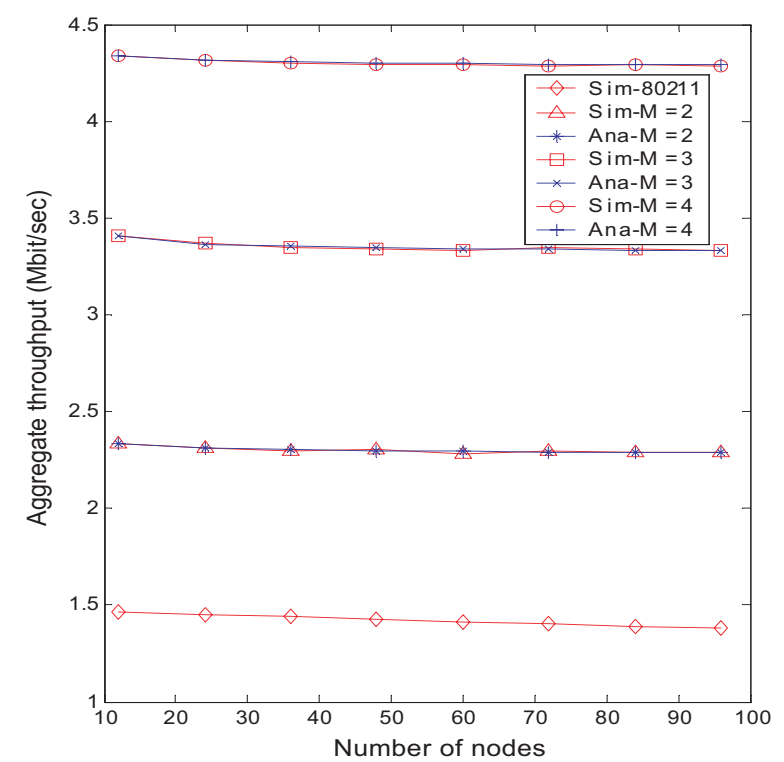

Fig. 6. Uplink saturated throughput with perfect beam-forming and even node-distribution.

number is 2,3 and 4 , respectively. In addition, simulation results validate the accuracy of our analytical model developed in Section VI.

Then we study the ideal non-saturated case in which we still assume beam-forming is perfect and nodes are evenly distributed. We assume all traffic goes from nodes to the access point. The packet arrival of each user follows a Poisson process. The average packet arrival rate ranges from 1 packet/user/sec to 45 packet/user/sec. The number of user is set to 24 . We use the stochastic approximation approach to adjust $T_{i n t}, T_{1}$ and $p$. As desired, these system parameters can quickly respond to traffic dynamics so that each uplink super-frame can be efficiently utilized; meanwhile, the average queue length $\bar{L}_{\max }$ can approach the target value $L_{\phi}$, which is set to 1 in our simulation. The traces of $\bar{L}_{\max }, T_{i n t}, T_{1}$ and $p$ are illustrated in Fig. 8, where the average packet arrival rate is 5 packet/user/sec and the beam-sector number is 3 . The throughput performance of the ideal non-saturated case is shown in Fig. 7. As shown in the figure, our system can significantly improve the non-saturated throughput.

After examining ideal cases with perfect beam-forming and even node-distribution, we evaluate the system performance under imperfect beam-forming and mobility. The antenna model simulated is shown in Fig. 9. Our antenna model abstracts beam-overlapping problem, multipath rich problem and back-lobe (side-lobe) effects as neighboring-beam-overlapping problem and all-beam-overlapping problem. The mobility model is the modified random-way point mobility model in which the max speed is $5 \mathrm{~m} / \mathrm{s}$, and the minimal speed is $0.001 \mathrm{~m} / \mathrm{s}$, and the average speed is $2.5 \mathrm{~m} / \mathrm{s}$. Simulation time of each scene is 500 s and each simulation result is averaged over 10 random cases. The transmission range is $250 \mathrm{~m}$ and the simulation field is a disk with $250 \mathrm{~m}$ radius. The beam-sector number is 3 and the total number of user is 24. Fig. 10 compares the throughput performance under different settings. Imperfect beam-forming does lead to a performance loss by reducing independent spatial reuse area. 


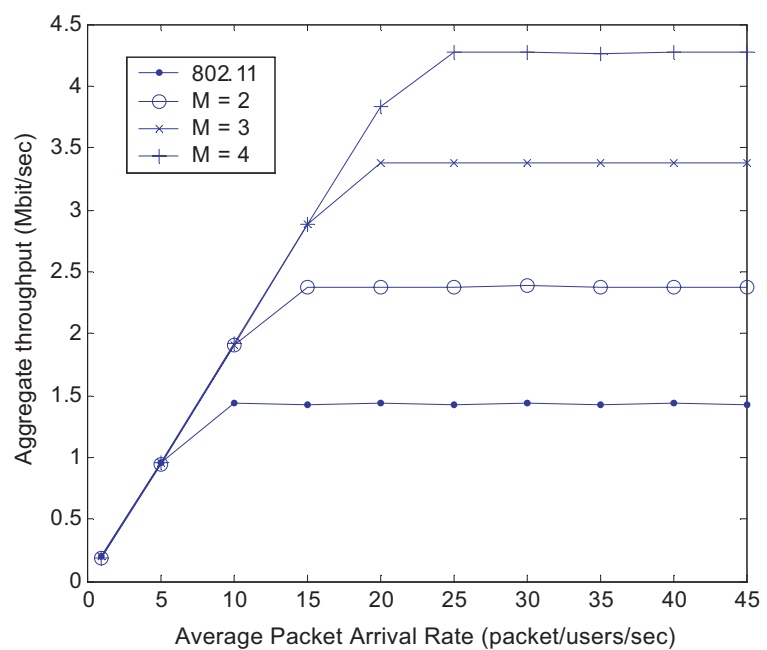

Fig. 7. Uplink non-saturated throughput with perfect beam-forming and even node-distribution.

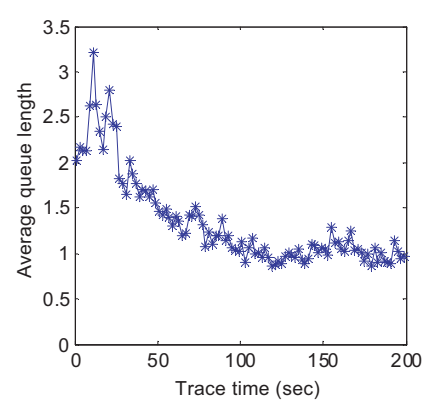

(a)

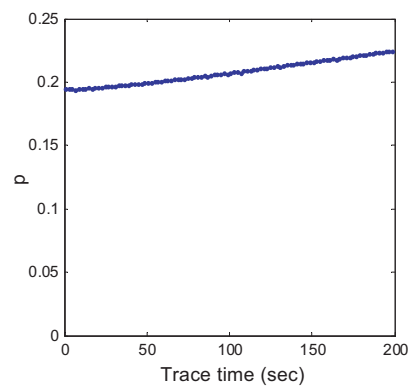

(c)

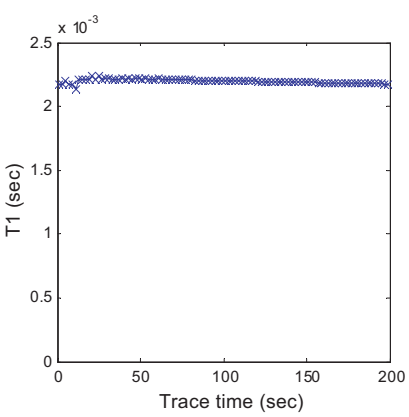

(b)

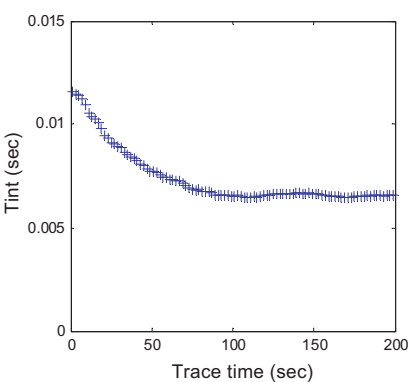

(d)
Fig. 8. Trace of system parameters: (a) $\bar{L}_{\max }$; (b) $T_{1}$; (c) $p$; (d) $T_{\text {int }}$

Mobility also affects the throughput performance by changing the node-distribution in each beam-sector. As compared to the case under perfect beam-forming and even node-distribution, the throughput with imperfect beam-forming and mobility is reduced by $22 \%$ approximately at the heavy traffic load. However, it still demonstrates the significant performance gain (about 90\%) in comparison with 802.11. In the extreme case, when only one client is served by the AP due to multipath, the performance could be worse than 802.11, as indicated in Fig. 10. The reason is that the access point initiated uplink medium access introduces non-trivial overhead by sending RTR frameby-frame. One way to leverage the worst case is switching to 802.11 operation mode, i.e., user initiated uplink medium access, when no spatial reuse can be exploited such that 802.11 equivalent performance can be guaranteed.

Our last simulation investigates the mobility effect on

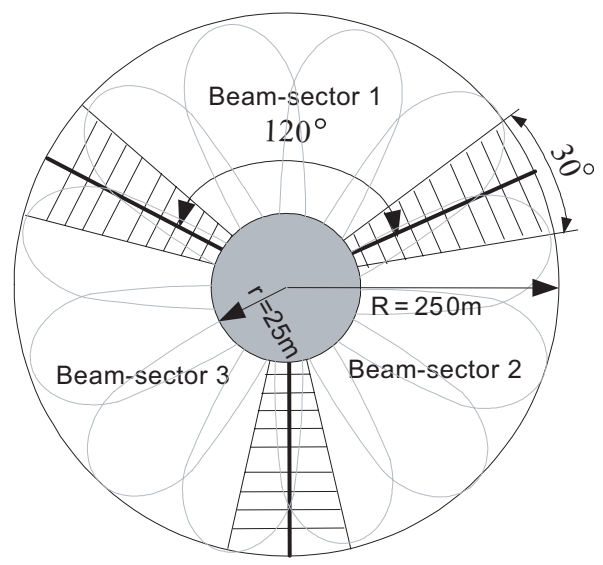

All-Beamoverlapping area

Neighboring-Beam overlapping area

Fig. 9. The simulated antenna model.

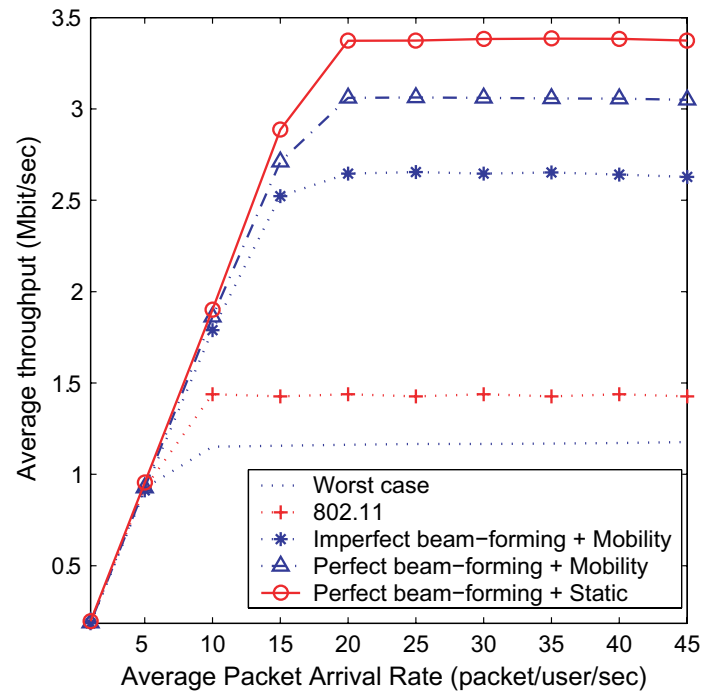

Fig. 10. Uplink throughput with imperfect beam-forming and mobility.

the performance of downlink medium-access. We use the same antenna model and mobility as the one in the last set of simulations. All the traffic is from the access point to mobile nodes and saturated. We apply the scheduling algorithm (choosing candidate receivers) at the access point such that as many independent nodes as possible can be selected for concurrent transmission given the throughput fairness is guaranteed. Multicast-omnidirectional-RTS is sent to request each candidate receiver to reply CTS. CTS is replied in sequence to confirm transmission and update beam-location information. In other words, the scheme characterized by Multicast-omnidirectional-RTS and sequential-CTS is evaluated. As shown in Fig. 11, our proposed downlink mediumaccess scheme is very robust to mobility when it increases from $1 \mathrm{~m} / \mathrm{s}$ to $10 \mathrm{~m} / \mathrm{s}$. Even with imperfect beam-forming and high mobility, our downlink throughput performance can still improve by about $120 \%$ over 802.11 .

\section{CONCLUSIONS}

In this paper, we proposed a CSMA/CA based medium access control protocol for wireless LANs with access point 


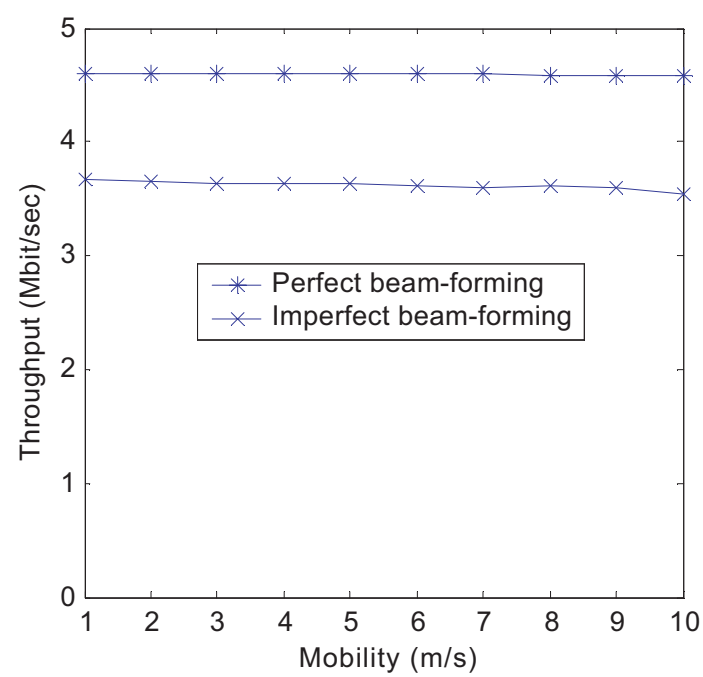

Fig. 11. Downlink throughput with imperfect beam-forming and mobility.

having multi-beam directional antennas. Our protocol efficiently utilizes spatial reuse by carefully addressing such challenging problems as the beam-synchronization problem, the beam-overlapping problem and the mobility issue. In addition, backward compatibility with IEEE 802.11 (DCF mode) is supported with minimal modification. We also present an analytical model to evaluate the performance of multibeam wireless LANs. Simulation results validate the analytical model and show that our scheme can significantly improve throughput, as compared to IEEE 802.11 MAC.

\section{REFERENCES}

[1] Y. Li, M. J. Feuerstein, and D. O. Reudink, "Performance evaluation of a cellular base station multibeam antenna," IEEE Trans. Veh. Tech., vol. 46, pp. 1-9, Feb. 1997.

[2] A. Swaminathan, D. L. Noneaker, and H. B. Russell, "The receiver blocking problem in a DS mobile ad hoc networks with directional antennas," in Proc. IEEE Military Communications Conference 2004.

[3] D. Lal, V. Jain, Q. Zeng, and D. P. Agrawal, "Performance evaluation of medium access control for multiple-beam antenna nodes in a wireless LAN," IEEE Trans. Parallel Distrib. Sys., vol. 15, no. 12, Dec. 2004.

[4] V. Gambiroza, B. Sadeghi, and E. Knightly, "End-to-end performance and fairness in multihop wireless backhaul networks," in Proc. of ACM MobiCom 2004.

[5] G. Xu, S.-Q. Li, "Throughput multiplication of wireless LANs for multimedia services: SDMA protocol design," in IEEE Global Telecommunications Conference (GLOBECOM '94), pp. 1326-1332.

[6] C. Sakr, T. D. Todd, "Carrier-sense protocols for packet-switched smart antenna base stations," in International J. Wireless Inf. Networks, vol. 7, no. 3, pp. 133-148, 2000.

[7] D. Lal, R. Toshniwal, R. Radhakrishnan, D. P. Agrawal, Jr., and J. Caffery, "A novel MAC layer protocol for space division multiple access in wireless ad hoc networks," in Proc. Eleventh International Conference on Computer Communications and Networks 2002, pp. 614-619.

[8] T. Ren, I. Koutsopoulos, and L. Tassiulas, "Efficient media access protocols for wireless LANs with smart antennas," in Proc. IEEE Wireless Communications and Networking (WCNC) 2003, vol. 2, pp. 1286-1290.

[9] G. Bianchi and I. Tinnirello, "Kalman filter estimation of the number of competing terminals in an IEEE 802.11 network," in Proc. IEEE INFOCOM 03, vol. 2, pp. 844-852.

[10] L. P. A. Robichaud, Signal Flow Graphs and Applications. Englewood Cliffs, New Jersey: Prentice-Hall, 1962.
[11] H. Zhai, Y. Kwon, and Y. Fang, "Performance analysis of IEEE 802.11 MAC protocol in wireless LAN," Wiley J. Wireless Commun. Moble Comput., vol. 4, pp. 917-931, 2004.

[12] H. Kushner and G. Yin, Stochastic Approximation Algorithms and Applications. New York: Springer-Verlag, 1997.

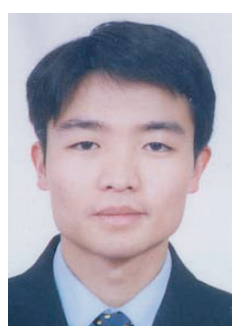

Jianfeng Wang ( $\left.\mathrm{S}^{\prime} 03-\mathrm{M}^{\prime} 06\right)$ received the B.E. and M.E. degrees in electrical engineering from Huazhong University of Science and Technology, China, in 1999 and 2002, respectively, and the Ph.D. degree in electrical engineering from University of Florida in 2006. From January 2006 to July 2006, he was a research intern in wireless standards and technology group, Intel Corporation. In October 2006, he joined Philips Research North America as a senior member research staff in wireless communications and networking department.

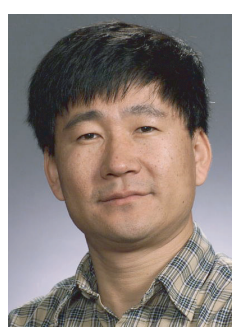

Yuguang Fang (S'92-M'94-S'96-M'97-SM'99) received a Ph.D degree in Systems and Control Engineering from Case Western Reserve University in January 1994, and a Ph.D degree in Electrical Engineering from Boston University in May 1997.

From June 1997 to July 1998, he was a Visiting Assistant Professor in Department of Electrical Engineering at the University of Texas at Dallas. From July 1998 to May 2000, he was an Assistant Professor in the Department of Electrical and Computer Engineering at New Jersey Institute of Technology. In May 2000, he joined the Department of Electrical and Computer Engineering at University of Florida where he got the early promotion to Associate Professor with tenure in August 2003 and to Full Professor in August 2005. He has published over 200 papers in refereed professional journals and conferences. He received the National Science Foundation Faculty Early Career Award in 2001 and the Office of Naval Research Young Investigator Award in 2002.

$\mathrm{He}$ is currently serving as an Editor for many journals including IEEE Transactions on Communications, IEEE Transactions on Wireless Communications, IEEE Transactions on Mobile Computing, and ACM Wireless Networks. He is also actively participating in conference organization such as the Program Vice-Chair for IEEE INFOCOM'2005, Program Co-Chair for the Global Internet and Next Generation Networks Symposium in IEEE Globecom'2004 and the Program Vice Chair for 2000 IEEE Wireless Communications and Networking Conference (WCNC'2000).

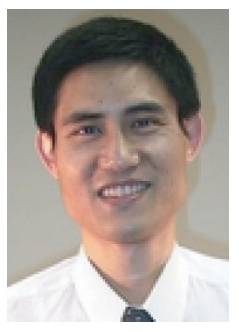

Dapeng Oliver Wu (S'98-M'04-SM06) received B.E. in Electrical Engineering from Huazhong University of Science and Technology, Wuhan, China, in 1990, M.E. in Electrical Engineering from Beijing University of Posts and Telecommunications, Beijing, China, in 1997, and Ph.D. in Electrical and Computer Engineering from Carnegie Mellon University, Pittsburgh, PA, in 2003.

Since August 2003, he has been with Electrical and Computer Engineering Department at University of Florida, Gainesville, FL, as an Assistant Professor. His research interests are in the areas of networking, communications, multimedia, signal processing, and information and network security. $\mathrm{He}$ received the IEEE Circuits and Systems for Video Technology (CSVT) Transactions Best Paper Award for Year 2001.

Currently, he is an Associate Editor for IEEE Transactions on Wireless Communications, IEEE Transactions on Circuits and Systems for Video Technology, IEEE Transactions on Vehicular Technology, and International Journal of Ad Hoc and Ubiquitous Computing. He is also a guest-editor for IEEE Journal on Selected Areas in Communications (JSAC), Special Issue on Cross-layer Optimized Wireless Multimedia Communications. He served as Program Chair for IEEE/ACM First International Workshop on Broadband Wireless Services and Applications (BroadWISE 2004); and as a technical program committee member of over 30 conferences. He is Vice Chair of Mobile and wireless multimedia Interest Group (MobIG), Technical Committee on Multimedia Communications, IEEE Communications Society. $\mathrm{He}$ is a member of the Best Paper Award Committee, Technical Committee on Multimedia Communications, IEEE Communications Society. 